\title{
Physical and Mechanical Research for Synthesis of Carbon Emocompatibly Coatings for Artificial Heart
}

\author{
Anna Voznesenskaya ${ }^{a}$, Valentin Morozov ${ }^{\mathrm{b}}$, and Alexey Zhdanov ${ }^{\mathrm{c}}$ \\ Vladimirst.Gorkyon87, Russian Federation
}

\begin{abstract}
To create an implant of the circulatory system using materials of different origin. The main requirements for such materials: rapid adaptation to the body and a long service life. Important characteristics are the hydrophobicity and surface roughness. Surface modification of materials by coating is the most common way to increase the functional properties. The study consisted in finding the perfect combination of the coating material and the technology of its application to the polyurethane product to increase its service life. In this study, we were applied two different methods of carbon coatings, which do not require heating of the polyurethane substrate: the method of laser deposition in a vacuum magnetron vacuum deposition method, and the pulverization of fine colloidal solution. Pulverization method applied to colloids with different powder: carbon nanotubes (CNT) and highly oriented pyrolytic graphite (HOPG), titanium oxide. Visualization of the surface produced on the optical, scanning electron and atomic force microscopy. Out of the samples, the most hydrophobic properties of the coated, fine colloidal solution HOPG caused by pulverization.
\end{abstract}

\section{Introduction}

The use of devices for circulatory support is one of the topical treatments for acute heart failure [1]. To create these devices attract a wide range of biocompatible materials (synthetic polymers, metals, ceramics, carbon, etc.) [2]. At the stage of synthetic biomaterials, simultaneously achieve optimal physical, mechanical and hemocompatible properties failed. Therefore, at present surface modification of biomaterials is the actual way to enhance blood compatibility products. The main requirements for implants - their rapid adaptation, as well as long service life, to avoid repeated operations. Such materials should not:

- provoke the formation of blood clots and blood clotting;

- have a negative effect on the protein components of blood;

- upset the electrolytic balance of blood;

- render toxic and allergic effects on the body;

- have carcinogenic effects;

- provoke the development of infection;

- lose functional properties within the prescribed period of operation [3].

Reaction to the implant is dependent on its surface properties: chemical composition, structure and morphology [4]. Therefore, methods for regulating the biological properties aimed at changing the physicochemical properties of the surface (chemical composition, degree of hydrophobicity, surface charge, and morphology, etc.) [5]. For hydrophobic surface is required to comply with the following conditions: 1) the surface roughness should be a combination of topography at the micro- and nanoscale; 2) the water contact angle should be more than $90^{\circ}$ [6].

For the manufacture of artificial hearts used biocompatible polymer - polyurethane. Polyurethane degraded in the living organism and there is a need to modify the surface by depositing a hemocompatible coating.

Based on the properties of the substrate (plasticity) coating should not form a continuous, brittle structure. Because when changed the geometry of the product (tension, compression, deformation) the coating will form crack, flake, peel off, to form pores.

\section{Methodology}

The study used as a substrate biocompatible polymer polyurethane "Vitur-TM0533-90"and material for the surface modification is of carbon since it is biologically stable than polyurethane, does not cause toxic and inflammatory reactions and will help to avoid degradation of the material, ensuring a good implant biocompatibility with blood plasma proteins and the living cells of the human body [4]. For completeness and comparison of the results of the study have been deposited several other biocompatible materials. On the surface of the polymer substrate, possibly the formation of nanostructured carbon, by thermodynamic and geometric parameters agree well with a certain type of

Corresponding author: ${ }^{\mathrm{a}} \underline{\text { 2obk@bk.ru}},{ }^{\mathrm{b}}$ vvm@vlsu.ru, ${ }^{\mathrm{c}}$ zhdanov@,vlsu.ru 
molecules of blood plasma proteins. For these reasons, when used as a modifier carbon coating can expect the formation of high-quality phase boundary polymer-blood [5].

Due to its low melting point $\left(\sim 160^{\circ} \mathrm{C}\right)$ of the polyurethane substrate should be used technology that does not require heating of the substrate: laser deposition [7], magnetron-vacuum deposition and spraying colloidal finely dispersed solute.

For coating of titanium and carbon has been used a method of laser ablation under vacuum. This method of forming films and coatings by condensation on the surface of the substrate products of the interaction of laser radiation with the target material in a vacuum. The laser beam focused on the target surface, which leads to destruction of its surface layer [8]. Material passes into the state of a plasma consisting of ions, atoms, clusters, and are deposit on a substrate to form a thin film [9].Application of carbon coating occurs using two laser systems: ytterbium fiber laser continuous PM-02-T (IPG IRE-Polus, Zelenograd) and millisecond laser system. The impact of continuous laser radiation on the graph (Figure 1) occurs within 5 minutes with the power of 70 $\mathrm{W}$, the pressure in the chamber was $4,6 \cdot 10-4 \mathrm{mB}$, the wavelength of $1030 \mathrm{~nm}$.

Sputtering target HOPG by pulsed laser was carried out with 4.5 Joules of energy, for 400 pulses, the wavelength of $1064 \mathrm{~nm}$, at a chamber pressure of 4,6 $10-4 \mathrm{mB}$. Application of titanium coating occurred along the same lines, was replaced only sputtering target: was carried out with $4 \mathrm{~J}$ energy, with the number pulses 200 , the wavelength $1030 \mathrm{~nm}$, at a pressure in the chamber 4 • $10-4 \mathrm{mB}$

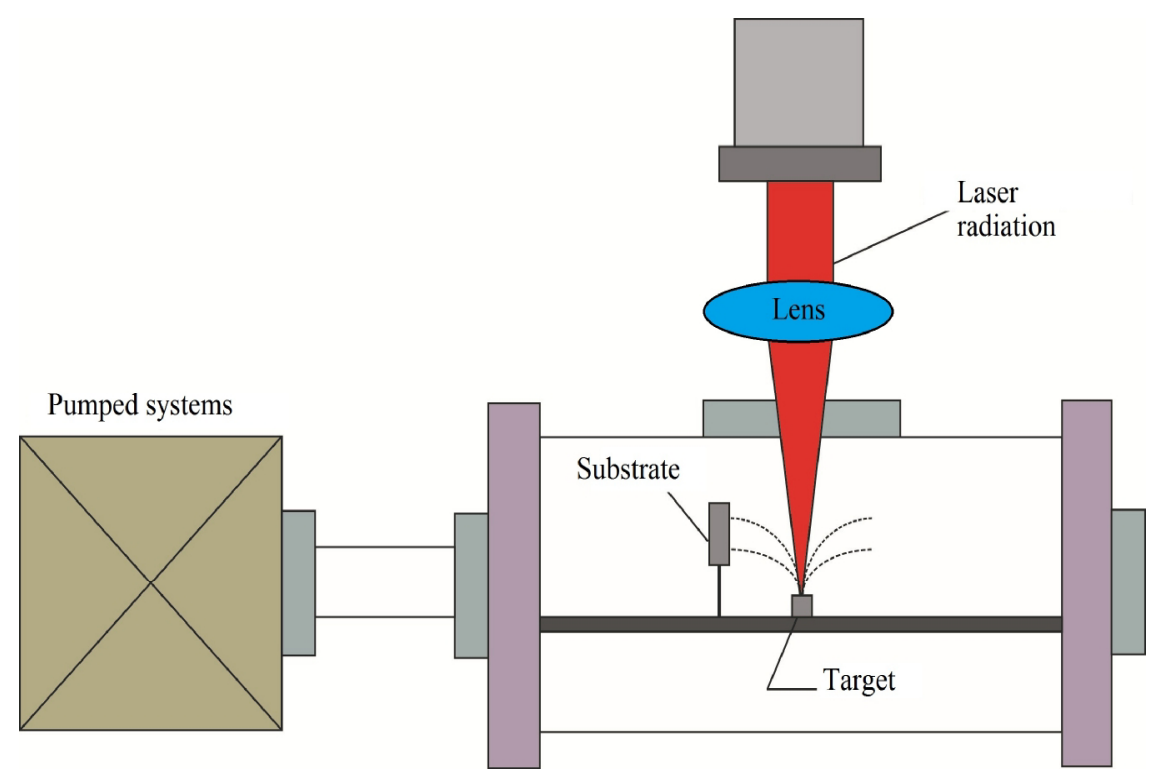

Figure 1. Scheme laser deposition in a vacuum.

For the preparation of the colloidal solution been selected few powders: highly oriented pyrolytic graphite (HOPG), carbon nanotubes (CNTs), titanium oxide. Used as a solvent dimethylformamide (C3H7NO).

Colloidal solutions for the application of the pulverization method of preparing two ways:

1) A solution prepared by laser ablation using a femtosecond laser TETA-10 with $\mathrm{Yb}: \mathrm{KGW}$ active medium ("Avesta", Troitsk). Femtosecond laser radiation was focused on a graphite target (Figure 2), beating the material particles in a liquid medium.

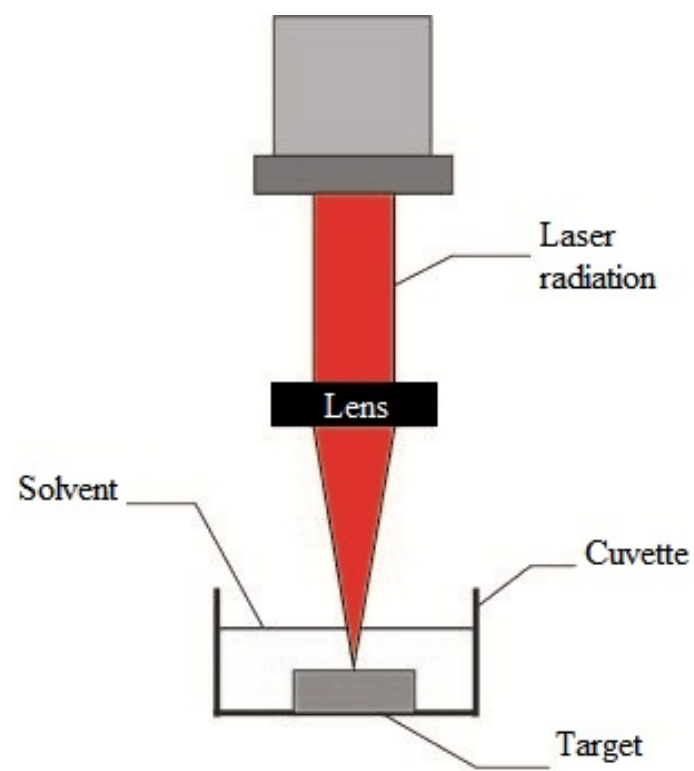

Figure 2. Scheme of laser ablation in liquids. 
2) A solution obtained by dissolving the powders in the liquid. Powders HOPG CNT and titanium oxide mixed with the solvent dimethylformamide 1:10. For sifting coarse graphite particles solution passed through a filter with meshes size of 150, 75, 35, 15 and 10 microns. For the deposition of the larger particles, within days solution stood in the flask standing vertically fixed. In the upper part of the vessel, the particle size was less than 1 micron.

The resulting solutions been sprayed on surface polyurethane.

\section{Results and Discussion}

1) Structural evaluation of coatings were performed by scanning electron microscopy on the device Quanta 200 3D (FEI Company, the Netherlands).

2) The roughness analyzed by nano-lab NTEGRA Spectra (NT-MDT, Russia, Zelenograd). The surface roughness of the deposited layers determined by atomic force microscopy (AFM). Presented roughness $\mathrm{Rz}$ calculated using the standard technique of 10 points as the arithmetic average of the absolute deviations of the height 5 of the highest and the deepest 5 points from the centerline of the surface profile[10]. Roughness coefficient calculated as follows:

$$
\mathrm{Rz}=\frac{\sum_{i=1}^{\mathrm{i}}|\mathrm{ypmi}|+\sum_{i=1}^{\mathrm{a}}\left|y_{\mathrm{w}}\right|}{\mathrm{s}}
$$

where $\mathrm{Rz}$ - average sum of absolute values of the heights of five maximum projection profile and the depth profile of the five largest cavities within the base line; ypmi - the height of the $i$ largest projection profile; yvmi - the depth of the i greatest depression profile.

3) Measure of the intensity of intermolecular interaction is a surface tension at the interface of water and substance. Than more hydrophilicity substance, the lower the surface tension. Hydrophilicity and hydrophobicity determined by contact angle wetting. This angle measured between the surface and the water drop. Determination wettability water been performed with an optical microscope, followed by image processing program Altami Studio, where it is possible to determine the geometric characteristics of the objects.

Graphite coating, applied continuous laser (2), laid down the hilly periodic structure (Figure 3). Angle wetting of 93o. Roughness coefficient was calculated according to the formula (1) and amounted to: $\mathrm{Rz}=1,79$ $\mu \mathrm{m}$. (Figure 4). Other coatings deposited by laser, get with cracks and defects.

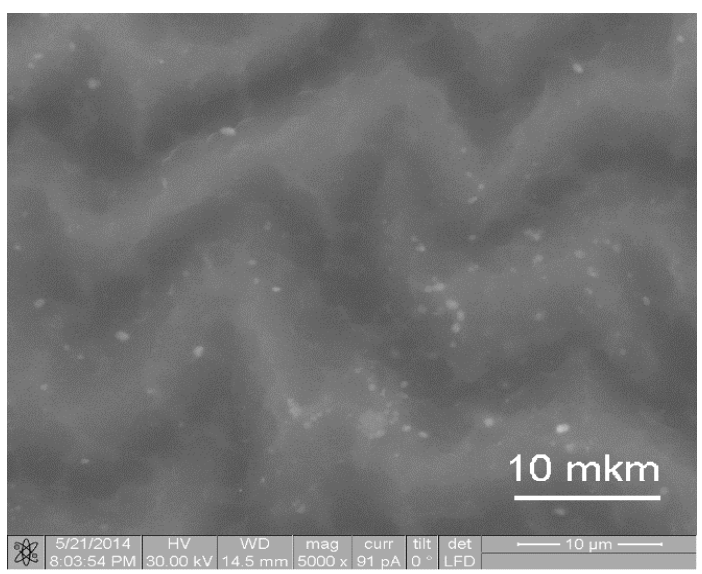

Figure 3. SEM image of HOPG coating (continuous laser radiation)

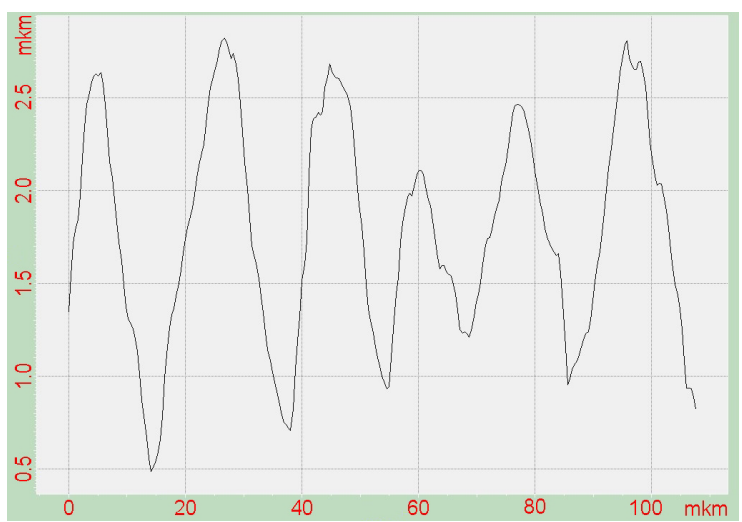

Figure 4. The roughness of the coating HOPG (continuous laser radiation)

When magnetron vacuum method sprayed, material lay chaotically, with an amorphous structure. Formation of clusters and grains make the material brittle. On the surface of the sample, there is a large amount of exfoliating flakes formed due to low adhesion of the coating to the substrate material. Particle size 5 to 25 microns, approximately rectangular, angle wetting titanium nitride $-130^{\circ}$, coefficient roughness could not be calculated due to abrupt changes in elevation, which threatened breaking of the probe.

HOPG coating (applying pulverization method), represents a granular structure, the particle size of 82 to $108 \mathrm{~nm}$ (Figure 5). Due to the solvent, the particles penetrate the surface layer of polyurethane, thereby providing high adhesion of the coating to the substrate. Due to this coating becomes elastic as polyurethane substrate. Thus received coating is does not exfoliate, lasting and hydrophobic. (Figure 6) 


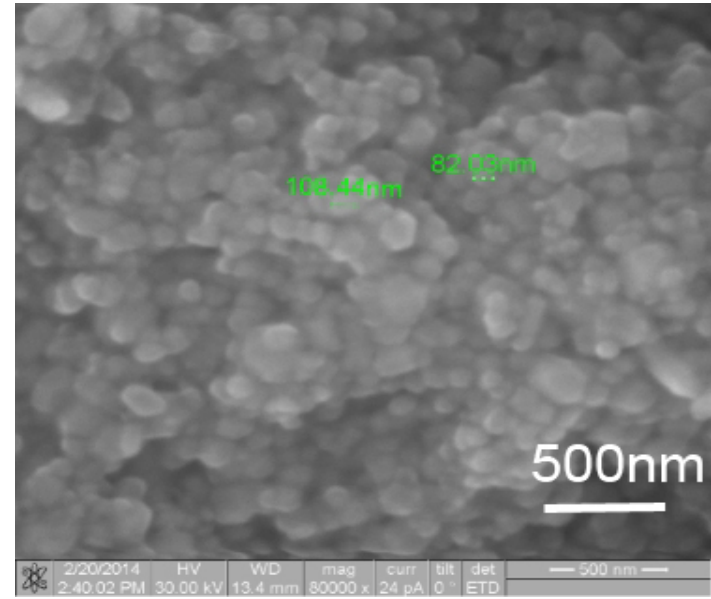

Figure 5. REM- cover image of graphite coating (pulverization application)

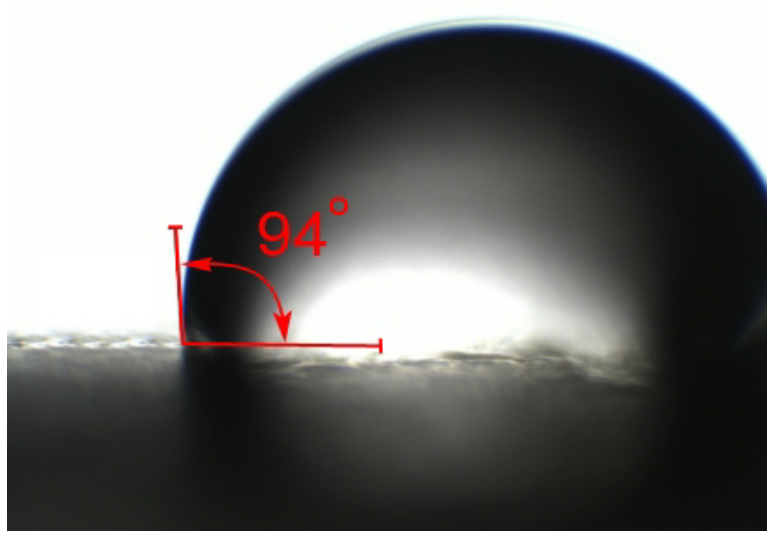

Figure 6. The angle wetting of graphite coating (pulverization application).

All results of the study shown in Table 1 .

Table 1. Key findings.

\begin{tabular}{lcc}
\hline & $\begin{array}{c}\text { Coefficientofroughness, } \\
\boldsymbol{R} \boldsymbol{z}[\mathbf{n m}]\end{array}$ & $\begin{array}{c}\text { Angle } \\
\text { wetting, } \\
\boldsymbol{\alpha}[\mathbf{d e g}]\end{array}$ \\
\hline $\begin{array}{l}\text { Polyurethane } \\
\text { substrate }\end{array}$ & 152 & 57 \\
& Laser depositionin a vacuum & \\
\hline $\begin{array}{l}\text { Graphite } \\
\text { (cwlaser) }\end{array}$ & 1790 & 87 \\
$\begin{array}{l}\text { Graphite (msec) } \\
\text { Titan (msec) }\end{array}$ & - & 71 \\
& 199 & 66 \\
\hline
\end{tabular}

\begin{tabular}{lcc}
\hline \begin{tabular}{l} 
Titaniumnitride \\
\multicolumn{1}{c}{ Pulverizationsprayingcolloidalsolution }
\end{tabular} & 50 \\
\hline $\begin{array}{lcc}\text { HOPG(laserablat } \\
\text { ion) }\end{array}$ & 25 & 61 \\
Titaniumoxide & & \\
CNT & 3480 & 63 \\
HOPG (powder) & 786 & 65 \\
& 192 & 94
\end{tabular}

\section{Conclusions}

Carbon coating (continuous laser irradiation in vacuum) and coating of graphite (pulverization coated colloidal solution), been obtained the most successful. We can assume that been obtained hemocompatible coating. These samples will be testing on the processes of interaction with blood.

The reported study was partially supported by RFBR, research project No. 13-08-01306 a.

\section{References}

L.V. Belyaev, A.V. Zhdanov, Advanced mechanical and materials, 96, 951-957 (2015)

S. L. Vasin, E.A. Abramovich, N.V. Perova, I. B. Rozanova, V. I. Sevastyanov, A. B. Schechte, Biocompatibility, 368(1999)

V. I. Sevastyanov, N. V. Perov, I. A. Dovizhik, Technology of living systems, 6, 10-19(2009)

A. P. Alekhin, A.G. Kirilenko, R.V. Lapshin, R.I. Romanov, A.A. Cigarev, Journal of Applied Chemistry, 76, 1536-1540 (2003)

A. P. Alekhin, Russian Journal of Nanotechnology, 5, 128-136 (2010)

L. N. Nikitin, Russian Chemical Journal, LII, 56-65 (2008)

G. K. Hyde, Biomedical materials, 4, 1-10(2009)

T. Akatsu, A. Kantrowitz, J.Biomed. Mater. Res., 1, 33-54 (1967)

R. Ebner, J. M. Lackner, W. Waldhauser, R. Major, E. Czsrnowska, R. Kustosz, P. Lack, Bulletin of the polish academy of science, 54, 167-173(2006)

I. A. Tsyganov, A. I. Pozdnyakov, E. Richter, M. F. Maytts, Bulletin of the Nizhny Novgorod University, NI Lobachevsky, 1, 52-56 (2007) 